\title{
Adoption of computerized tomography perfusion imaging in the diagnosis of acute cerebral infarct under optimized deconvolution algorithm
}

\author{
Bo Fang ${ }^{1}$, Hongjiang Zhai ${ }^{2}$
}

\begin{abstract}
Objectives: To explore the significance of the hemodynamic parameters of Computerized Tomography Perfusion Imaging (CTPI) under the deconvolution optimization algorithm for the diagnosis and treatment of patients with acute cerebral infarct $(\mathrm{ACl})$.

Methods: A hundred and ten patients with ACl from December 2018 to September 2019 were selected for research, and CTPI was performed before and after Edaravone injection treatment. Then, the CTPI deconvolution algorithm based on the weighted adaptive (WA) total variation (TV) (WA-TV) optimization was constructed, which was compared with tensor total variation (TTV) and Motion-adaptive sparse parity (MASP). Brain Perfusion 4.0 was applied to obtain the relative time to peak (rTTP), the relative transit time of mean ( $\mathrm{rMTT}$ ), relative cerebral blood volume ( $\mathrm{rCBV})$, and relative cerebral blood flow ( $\mathrm{rCBF}$ ) of the core infarction area $(\mathrm{ClA})$ and penumbra ischemic $(\mathrm{PI})$.

Results: In four parameters of rTTP, rMTT, rCBV, and CBF, the peak signal to noise ratio (PSNR) of the WA-TV algorithm was higher than the MSAP and TTV algorithms, while the Mean Square Error (MSE) and Mean Absolute Error (MAE) were lower than MSAP and TTV algorithms $(P<0.05)$; the parameters of rCBV (71.56 \pm 9.87$), \mathrm{rCBF}(43.17 \pm 7.06)$ of the $\mathrm{CIA}$ before treatment were higher than $\mathrm{PI}(23.66 \pm 7.22 ; 18.37 \pm 3.99)$, rMTT $(124.83 \pm 9.73)$ and rTTP $(122.57 \pm 7.41)$ were lower than the PI $(183.17 \pm 10.16) ; 150.74 \pm 9.74)(P<0.05)$. After treatment, the $\mathrm{rCBV}$ and $\mathrm{rCBF}$ of PI were higher than before treatment, and rMTT and rTTP were lower than before treatment $(P<0.05)$, and there was no obvious difference in $r C B V, r C B F, r M T T$, and $r T T P$ before and after treatment in the CIA $(P>0.05)$.

Conclusion: Compared with TTV and MASP, the WA-TV algorithm performs better in noise reduction and artifact reduction. The CTPI parameters of $\mathrm{rCBV}, \mathrm{rCBF}, \mathrm{rMTT}$, and rTTP are all important indications for the diagnosis of $\mathrm{PI}$ and $\mathrm{ACl}$.
\end{abstract}

KEYWORDS: Deconvolution optimization algorithm; Computerized Tomography Perfusion Imaging; Core infarct area; Acute cerebral infarct; Penumbra ischemic.

doi: https://doi.org/10.12669/pjms.37.6-WIT.4884

How to cite this:

Fang $B$, Zhai $H$. Adoption of computerized tomography perfusion imaging in the diagnosis of acute cerebral infarct under optimized deconvolution algorithm. Pak J Med Sci. 2021;37(6):1687-1692. doi: https://doi.org/10.12669/pjms.37.6-WIT.4884

This is an Open Access article distributed under the terms of the Creative Commons Attribution License (http://creativecommons.org/licenses/by/3.0), which permits unrestricted use, distribution, and reproduction in any medium, provided the original work is properly cited.

\section{Correspondence:}

Hongjiang Zhai,

Department of Neurology,

Lu'an People's Hospital Affiliated to Anhui Medical University,

Lu'an, 237005,

Anhui Province, China.

E-mail: zhaihongjiang_zhj@yeah.net

* Received for Publication:

* Revision Received:

* Final Revision Accepted:
April 4, 2021

June 12, 2021

July 17, 2021

\section{INTRODUCTION}

ACI may occur if abnormal objects (solid, liquid, gas) enter the cerebral arteries or the neck arteries, block blood flow, and soften or even necrotize the brain tissues. ${ }^{1,2}$ The current clinical treatments for ACI include general treatment, thrombolytic therapy, and drug therapy. The general treatment nurses the patient's skin, mouth, respiratory tract, and urine. Thrombolytic therapy adopts 
recombinant tissue-type plasminogen activator to treat patients with intravenous thrombolysis. Medications include mannitol, heparin, and Edaravone. ${ }^{3-5} \mathrm{CT}$ can check the lesions of patients with ACI, with low cost, fast examination, and non-invasiveness. CTPI can check the brain with contrast agent injection, ${ }^{4-7}$ providing help for the clinical development of personalized thrombolytic therapy. Deconvolution can calculate various myocardial perfusion hemodynamic parameters, including myocardial blood flow and myocardial blood volume, to assess myocardial ischemia. ${ }^{8-10}$

Hence, the CTPI deconvolution algorithm based on the WA-TV optimization was constructed to compare with the TTV and MASP algorithms. CTPI was performed before and after the Edaravone injection treatment on 110 cases with $\mathrm{ACI}$, and the clinical value of CTPI was evaluated through comparison with the parameters of rTTP, rMTT, $\mathrm{rCBV}$, and $\mathrm{rCBF}$ of the CIA and the PI.

\section{METHODS}

One hundred ten patients with ACI admitted to the hospital from December 2018 to September 2019 were selected and performed CTPI before and after Edaravone injection treatment. The study was approved by the medical ethics committee of the hospital, and the patients and their families understood the study and signed an informed consent form.

Inclusion criteria: I. Patients with the first onset; II. Patients with complete clinical data; III. Patients who have not received thrombolytic therapy; IV. Patients with non-responsible low-density lesions screened by CT scan; V. Patients with clear onset time.

Exclusion criteria: I. Patients younger than 18 years; II. Patients who were unconscious and unable to cooperate with the examination; III. Patients with a history of epilepsy; IV. Patients who were allergic to iodine-containing contrast media; $\mathrm{V}$. Those who had received anticoagulant treatment.

A 128-slice spiral CT scanner of General Electric (GE) can examine patients. The contrast agent was Iopromide Injection 300 (Iodine Concentration $300 \mathrm{mg} / \mathrm{mL}$ ) of Bayer Pharmaceuticals. Here, a high-pressure syringe was adopted to inject patients with $50 \mathrm{~mL}$ of Iopromide into the elbow vein at a rate of $5 \mathrm{~mL} / \mathrm{s}$, and the patients are scanned from head to foot. A total of 20 cycles are scanned to obtain a time-dose-curve (TDC). The scanning parameters were tube voltage $125 \mathrm{kv}$, tube current $110 \mathrm{~mA}$, layer thickness $6 \mathrm{~mm}$, matrix
$512 \times 512$, and field of view $240 \times 240 \mathrm{~mm}$. After the CT scan, the perfusion image was sent to the Brain Perfusion 4.0 perfusion analysis software for the perfusion imaging parameters, namely, CBV, CBF, mean transit time (MTT), and time to peak (TTP). Then, according to the size of the CIA and the PI zone, the relative ratio of the affected side and the uninfected side of the abnormal perfusion area was obtained by mirror line symmetry, including rTTP, rMTT, rCBV, and rCBF.

Construction of deconvolution algorithm based on WA-TV optimization. First, the tracer dilution theory was introduced to construct a myocardial $\mathrm{CT}$ perfusion convolution model, that is, the arterial input function was used to convolve the blood flow scale function to obtain the tissue contrast agent concentration, the arterial input function was set as $\mathrm{B}$, and the blood flow scaling function was set as G. Then, the convolutional discrete matrix of the region of interest of the entire organization can be expressed as follows.

$$
C=B G(1)
$$

In which, $C$ was the concentration of tissue contrast agent, $C=\left[\mathrm{c}_{1}, \cdots, \mathrm{c}_{\mathrm{N}}\right], \mathrm{G}=\left[\mathrm{g}_{1}, \cdots, \mathrm{g}_{\mathrm{N}}\right], N$ was the number of voxels in the region of interest. Then, the regularization method can obtain a stable and accurate blood flow scale function, as shown in equation (2).

$$
G=\underset{g}{\arg \min }\|B G-C\|_{2}^{2}+R(\mathrm{G})
$$

Equation (2) was the myocardial CT perfusion convolution model, $R(\mathrm{G})$ represented the regularization term, and $G$ represented the blood flow scaling function. Using regularization to reduce the noise of CT perfusion images was a commonly used method, but the traditional TV has obvious shortcomings, which can cause image texture results to be lost due to step artifacts. To solve this problem, the WA-TV regularization method was introduced, and it performed adaptive adjustments according to the local image intensity to ensure the completeness of the image edge detail information, which was set as WA-TV. The objective function was as follows.

$$
G=\underset{g}{\arg \min }\|B G-C\|_{2}^{2}+\|G\|_{W A-T V}(3)
$$

$\|G\|_{W A-T V}$ represented the prior term of WA-TV regularization, which can be defined as follows.

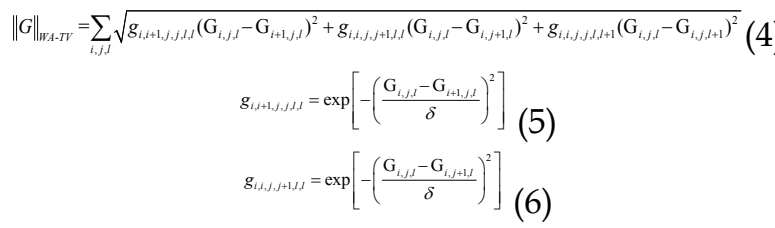




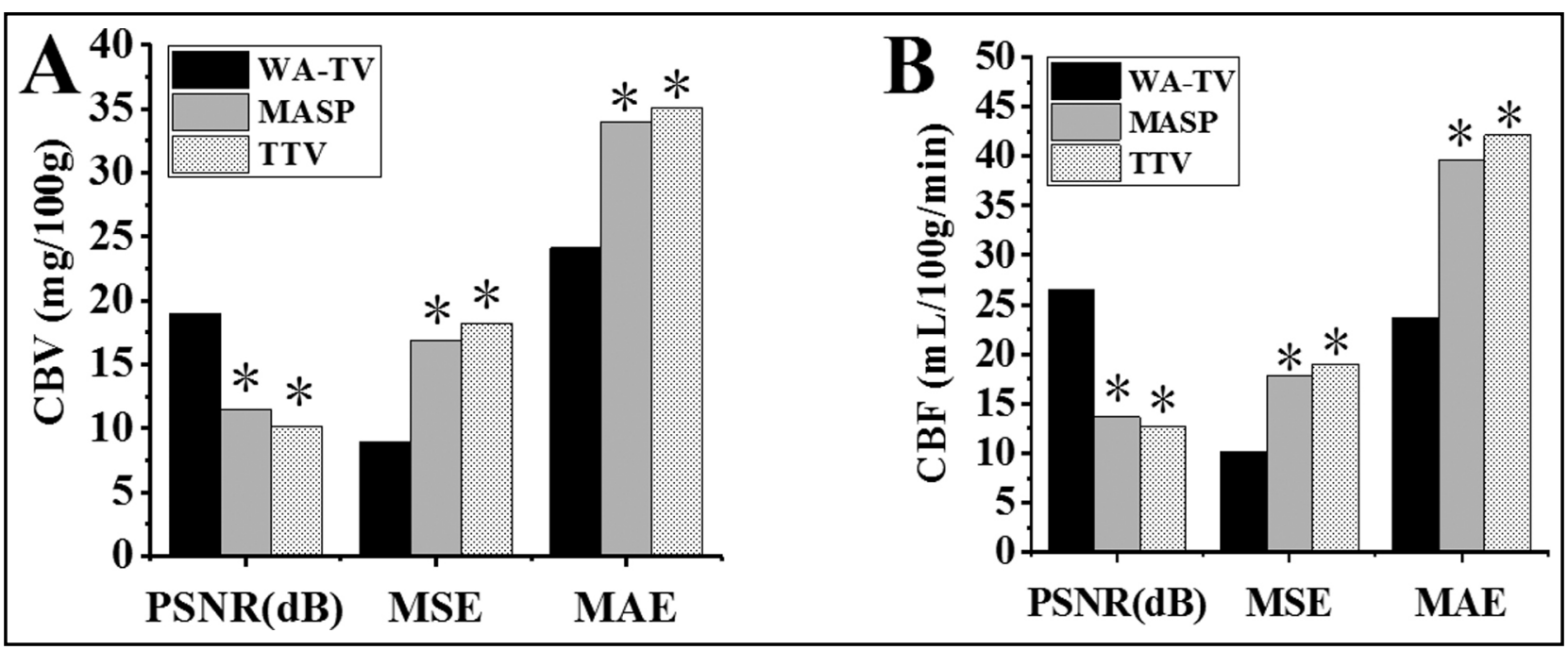

Fig.1: Performance of the three algorithms under different CT perfusion parameters. A was CBV; B was CBF; * indicated that the difference was obvious compared with the WA-TV $(\mathrm{P}<0.05)$.

$$
g_{i, j, j, t, 1+1}=\exp \left[-\left(\frac{\mathrm{G}_{i, j, i}-\mathrm{G}_{i, j, j+1}}{\delta}\right)^{2}\right](7)
$$

$\delta$ represented the scale factor for adjusting the iteration intensity, which was sensitive to changes in local voxel intensity. Then, the Iterative ShrinkageThresholding Method was introduced to optimize the WA-TV regularization prior terms, which can be expressed as follows.

$$
\begin{gathered}
G_{s}=\underset{g}{\arg \min }\|B G-C\|_{2}^{2} \text { with } \\
G=\underset{g}{\arg \min } \frac{1}{2}\left\|G-G_{s}\right\|^{2}+\|G\|_{W D-T V}
\end{gathered}
$$

If $G_{s}$ was the solution of the nth iteration, then the equation below was obtained.

$$
G_{s}=\bar{G}^{n}-r^{n+1} B^{T}\left(\mathrm{~B} \bar{G}^{n}-C\right)(10)
$$

In which, $\quad r^{n+1}=\frac{H^{T} H}{(\mathrm{BH})^{\mathrm{T}}(\mathrm{BH})}, \quad H \triangleq B\left(\mathrm{BH}^{\mathrm{n}}-\mathrm{C}\right)$. The above was the deconvolution algorithm based on WA-TV optimization. Evaluation indicators of the algorithm proposed. PSNR, MSE, and MAE can evaluate the performance of the WA-TV algorithm constructed, and TTV and MASP were introduced for comparison.

$$
\begin{array}{r}
\mathrm{PSNR}=10 \log _{10}\left[\frac{M A X^{2}\left(\mathrm{X}_{\text {ture }}\right)}{\sum_{n=1}^{O}\left(\mathrm{X}(\mathrm{n})-X_{\text {ure }}(\mathrm{n})^{2} /(Q-1)^{2}\right.}\right] \\
M S E=\frac{100}{\bar{X}_{\text {ture }}} \sqrt{\frac{1}{Q-1} \sum_{n=1}^{o}\left(\mathrm{X}(\mathrm{n})-X_{\text {ture }}(\mathrm{n})^{2}\right)} \\
M A E=\frac{100}{Q} \sum_{n=1}^{Q}\left|\frac{\mathrm{X}(\mathrm{n})}{X_{\text {ture }}(\mathrm{n})}-1\right|(13)
\end{array}
$$

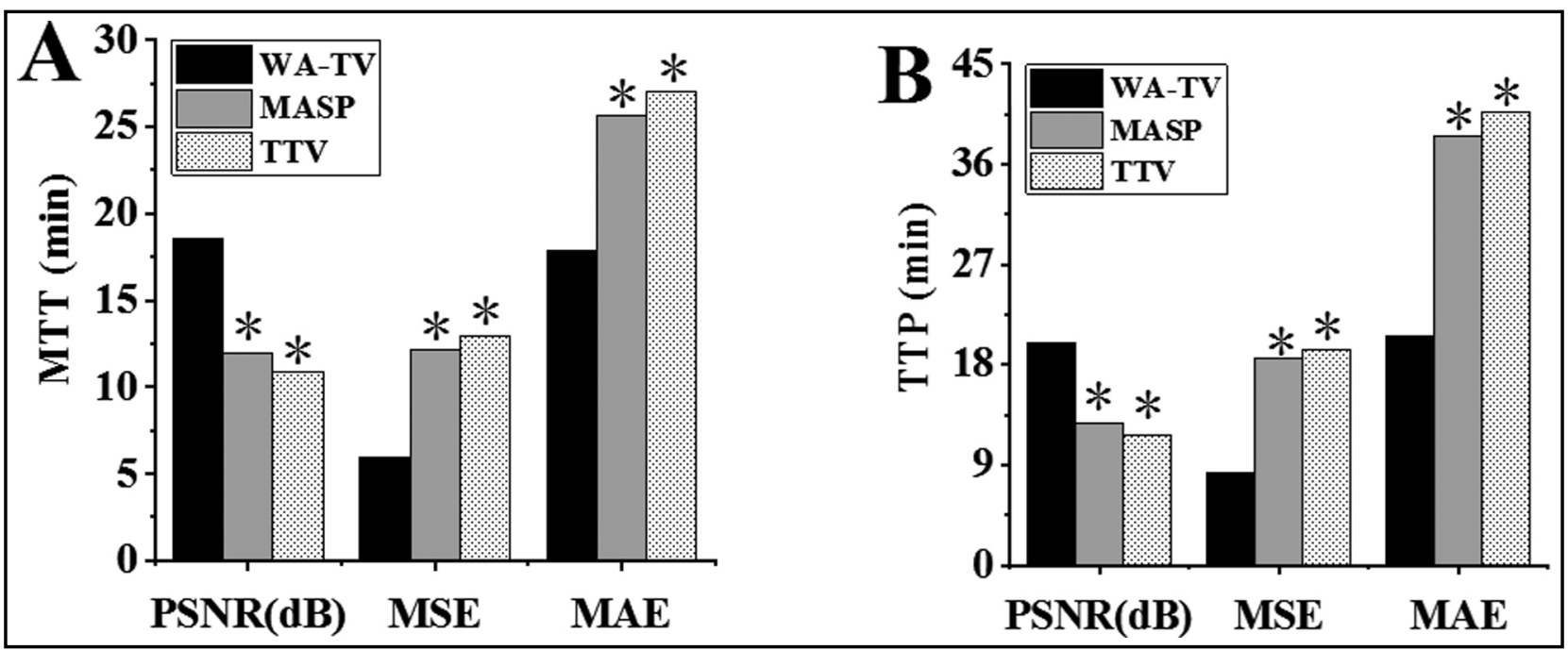

Fig.2: Performance of the three algorithms under different CT perfusion parameters. A was MTT; B was TTP. * indicated that the difference was obvious compared with the WA-TV $(\mathrm{P}<0.05)$. 


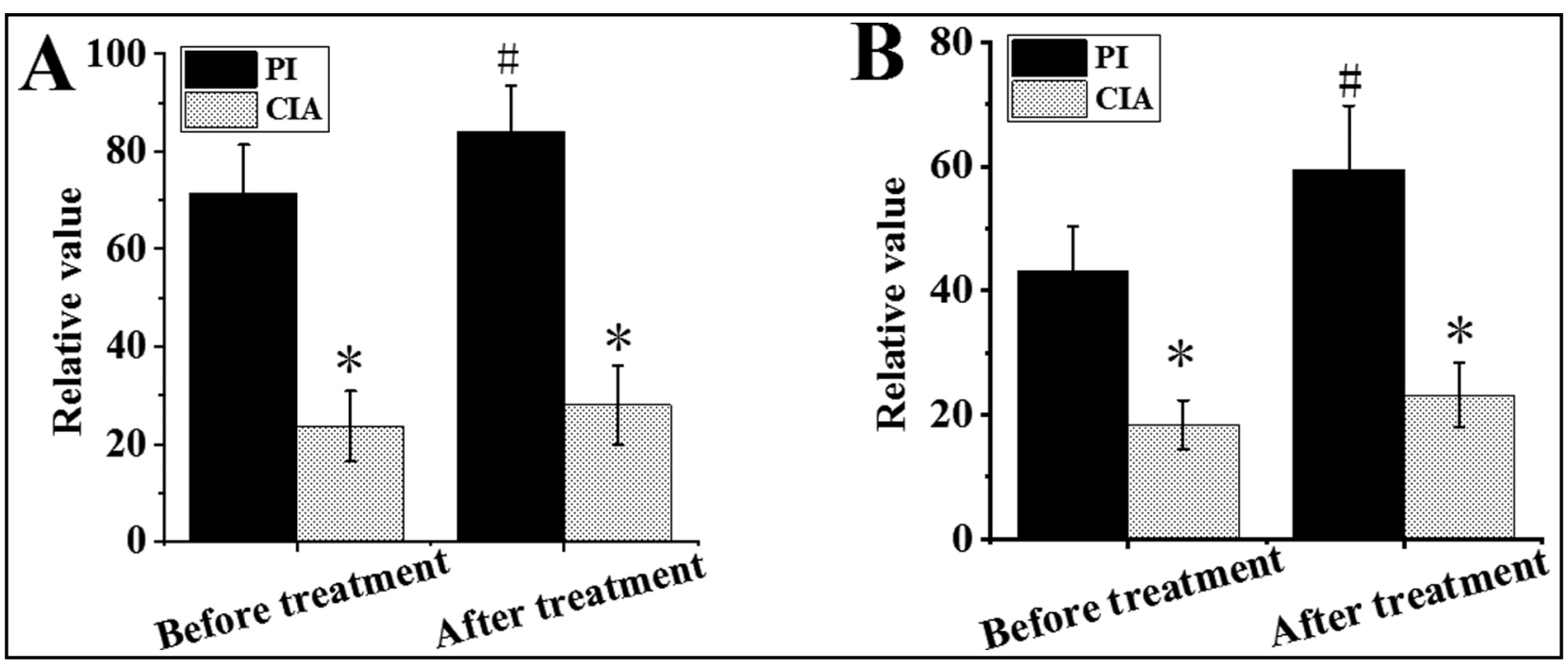

Fig.3: Comparison of relative values of PI and CIA parameters before and after treatment (rCBV and rCBF). A was rCBV; B was rCBF; * indicated that the difference was obvious compared with PI $(\mathrm{P}<0.05)$; \# indicated that the difference was obvious compared with that before treatment $(\mathrm{P}<0.05)$.

IX represented the pixel value of the area of interest, $X_{\text {ure }}$ represented the pixel value of the area of interest of the standard dose of contrast agent, $\bar{X}_{\text {true }}$ represented the average value of $X_{\text {ture }}$, and $Q$ represented the number of pixels.

Statistical Analysis: SPSS19.0, mean \pm standard deviation $(\mathrm{x} \pm \mathrm{s})$, and the percentage (\%) were adopted to process the count data. The comparison of PSNR, MSE, and MAE of WA-TV, MSAP, and TTV algorithms adopted t-test. The relative values of parameters in the PI and CIA before and after treatment were compared through variance. With $P<0.05$, the difference was statistically significant.

\section{RESULTS}

Table-I showed that the proportion of male patients was slightly higher than that of females; patients older than 50 years was the highest; patients with body mass index of 27-29.9 was the highest; and patients with diabetes, hypertension, and hyperlipidemia were higher than those without them, and the proportions of patients with or without a smoking history didn't differ much, neither did patients with or without coronary heart disease.

Performance of the three algorithms under different $\mathrm{CT}$ perfusion parameters. In Fig. $1 \& 2$, in the parameters of CBV, CBF, MTT, and TPP, the PSNR of the WA-TV algorithm was higher than that of the MSAP and TTV algorithms $(P<0.05)$; the MSE and MAE of the WA-TV algorithm were lower than the MSAP and TTV algorithms $(P<0.05)$.
Table-I: Basic data of patients.

\begin{tabular}{lccc}
\hline Variable & Classification & $\begin{array}{c}\text { Sample size } \\
\text { (person) }\end{array}$ & $\begin{array}{c}\text { Proportion } \\
(\%)\end{array}$ \\
\hline Gender & Male & 59 & 53.64 \\
& Female & 51 & 46.36 \\
Age (year) & $<=50$ & 11 & 10.00 \\
& $50-60$ & 23 & 20.91 \\
& $60-70$ & 44 & 40.00 \\
Body mass & $>=70$ & 32 & 29.09 \\
index & $<23.9$ & 18 & 16.21 \\
& $24-26.9$ & 29 & 26.15 \\
Diabetes & $27-29.9$ & 44 & 40.28 \\
Smoking & $>=30$ & 19 & 17.36 \\
history & With & 70 & 63.38 \\
& Without & 40 & 36.62 \\
Hypertension & With & 58 & 53.18 \\
& Without & 52 & 46.82 \\
Hyperlipidemia & With & 66 & 60.39 \\
Coronary Heart & Without & 44 & 39.61 \\
Disease & With & 71 & 64.28 \\
\hline & Without & 39 & 35.72 \\
& Without & 59 & 54.02 \\
& & & 45.98 \\
\hline
\end{tabular}




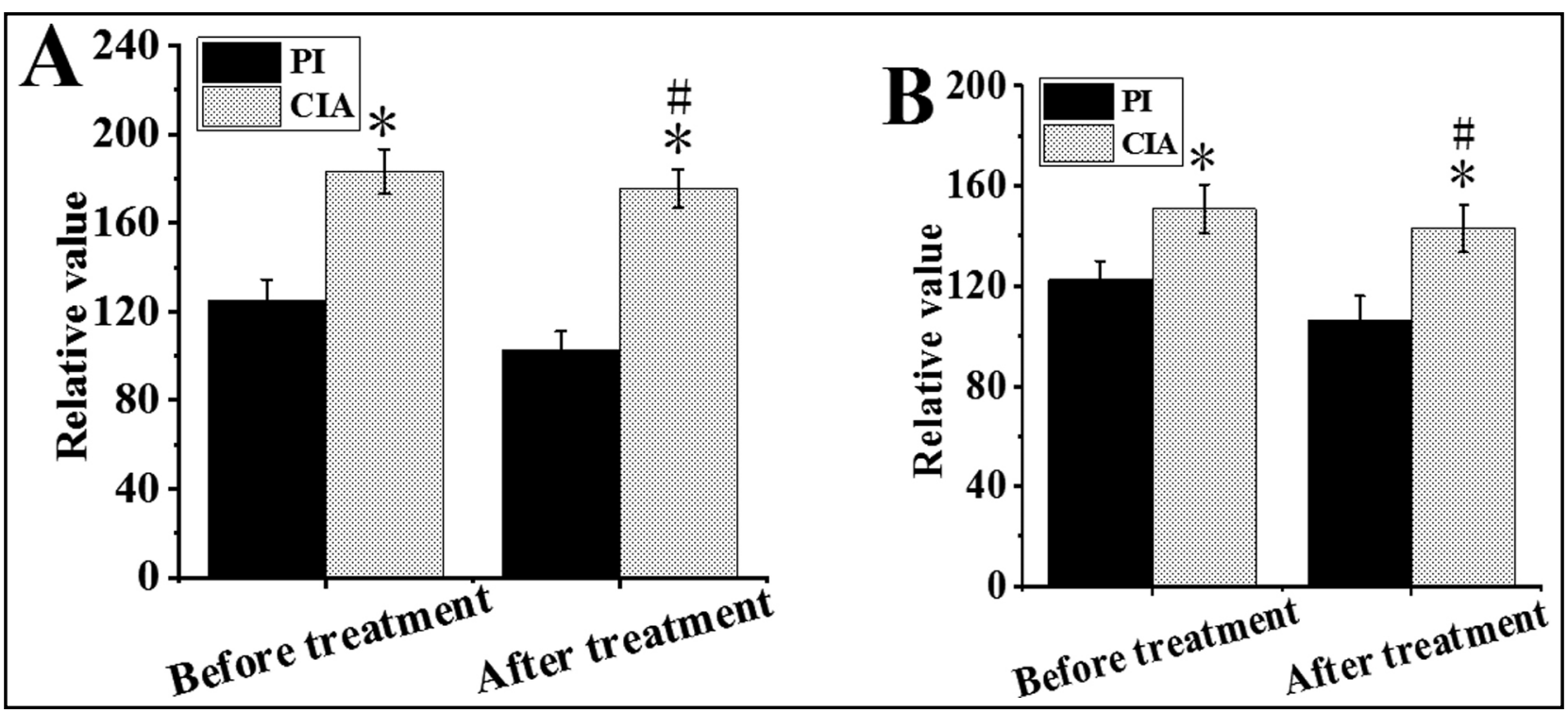

Fig.4: Comparison of relative values of PI and CIA parameters before and after treatment (rMTT and rTTP). A was the relative value of rMTT; B was the relative value of rTTP; * indicated that the difference was obvious compared with PI

$(\mathrm{P}<0.05)$; \# indicated that the difference was obvious compared with that before treatment $(\mathrm{P}<0.05)$.

Comparison of relative values of PI and CIA parameters before and after treatment. As shown in Fig.3 \& 4, the rCBV and $\mathrm{rCBF}$ in PI after treatment were higher than before treatment, and rMTT and rTTP were lower than before treatment $(P<0.05)$; the difference of $\mathrm{rCBV}, \mathrm{rCBF}, \mathrm{rMTT}$, and rTTP before and after treatment in the CIA was not obvious $(P>0.05)$.

\section{DISCUSSION}

The results showed that the WA-TV algorithm proposed in this study had a higher PSNR in contrast to the TTV and MASP algorithms after the CTPI images were processed. The PSNR index is a quantitative index used to evaluate the effect of image reconstruction. The larger the PSNR value, the closer the resolution of the reconstructed image to the original image. ${ }^{11-13}$ Therefore, it suggested that the resolution of the CTPI image reconstructed by the WA-TV algorithm proposed in this study was higher, and the reconstruction result was significantly better than that of the TTV and MASP algorithms.

Subsequently, the CTPI images processed by the WA-TV algorithm was applied to compare and analyze the changes in the brain tissue structure of patients with ACI before and after treatment. The indicators used to evaluate the effect of the indicators included CBV, CBF, MTT, and TTP. CBV refers to the VOLUME of blood through a certain cross-section of cerebrovascular in a unit time, which can reflect the damage of brain tissue. ${ }^{14-15} \mathrm{CBF}$ refers to the total amount of blood contained in cerebral blood vessels (including cerebral arteries, arterioles, and capillaries), and it has important guiding value for the clinical treatment of patients with acute cerebral infarction. ${ }^{16,17}$. MTT means the average time for blood to pass through the vasculature of a specific brain area. ${ }^{18,19}$ TTP refers to the time required for the contrast agent to reach the maximum concentration of the brain tissue. ${ }^{20}$ The results of this study revealed that $C B V$ and $\mathrm{CBF}$ were much higher in patients with ACI before treatment, while MTT and TTP values were greatly lower. It showed that treatment can dramatically improve the perfusion state of ischemic brain tissue in patients with $\mathrm{ACI}$, and reduce the damage to the brain tissue and nerve function of patients.

\section{CONCLUSION}

Here, the CTPI deconvolution algorithm was constructed based on the WA-TV optimization, the performance was compared with TTV and MASP, and CTPI scanned patients with ACI. Consequently, compared with TTV and MASP, the WA-TV algorithm has better noise reduction and artifact reduction. The CTPI parameters of rCBV, rCBF, rMTT, and rTTP are all important indications for the diagnosis of PI and ACI. 
Limitations of the study: The sample size is small, it has not been compared with normal brain tissue. Later, an enlarged patient sample should be considered to further explore the effectiveness of the deconvolution algorithm. In short, the results provide a reference for the application of CTPI parameters based on the deconvolution algorithm in the diagnosis of ACI.

\section{REFERENCES}

1. Jin X, Zou Y, Zhai J, Liu J, Huang B. Refractory Mycoplasma pneumoniae pneumonia with concomitant acute cerebral infarction in a child: A case report and literature review. Medicine. 2018;97(13):e0103. doi: 10.1097/MD.0000000000010103

2. Nakamura Y, Nakajima H, Kimura F, Unoda K, Arawaka S. Preventive Effect of Cilostazol on Pneumonia in Patients with Acute Cerebral Infarction. J Stroke Cerebrovasc Dis. 2018;27(9):2354-2359. doi: 10.1016/j. jstrokecerebrovasdis.2018.04.024

3. Landais A, Chaumont H, Dellis R. Thrombolytic Therapy of Acute Ischemic Stroke during Early Pregnancy. J Stroke Cerebrovasc Dis. 2018;27(2):e20-e23. doi: 10.1016/j. jstrokecerebrovasdis.2016.12.010

4. Phipps MS, Jastreboff AM, Furie K, Kernan WN. The diagnosis and management of cerebrovascular disease in diabetes. Curr Diab Rep. 2012;12(3):314-323. doi: 10.1007/ s11892-012-0271-x

5. Yan Z, Fu B, He D, Zhang Y, Liu J, Zhang X. The relationship between oxidized low-density lipoprotein and related ratio and acute cerebral infarction. Medicine. 2018;97(39):e12642. doi: 10.1097/MD.0000000000012642

6. Xu M, He XY, Huang P. The Relationship between the Mean Platelet Volume and Carotid Atherosclerosis and Prognosis in Patients with Acute Cerebral Infarction. Biomed Res Int. 2020;2020:6685740. doi: 10.1155/2020/6685740

7. Liang Y, Wu J, Liu J, Liu H, Chen J. The Clinical Implications of Thrombelastography in the Diagnosis of Acute Cerebral Infarction. Clin Lab. 2018;64(1):147-152. doi: 10.7754/Clin. Lab.2017.170803

8. Guan Y, Wang P, Wang Q, Li P, Zeng J, Qin P, et al. Separability of Acute Cerebral Infarction Lesions in CT Based Radiomics: Toward Artificial Intelligence-Assisted Diagnosis. Biomed Res Int. 2020;2020: 8864756. doi: 10.1155/2020/8864756

9. Liu YX, Cao QM, Ma BC. Pathogens distribution and drug resistance in patients with acute cerebral infarction complicated with diabetes and nosocomial pulmonary infection. BMC Infect Dis. 2019;19(1):603. doi: 10.1186/ s12879-019-4142-9

10. Zhang Q, Ba C, Zhang M, Liu Z, Shi B, Qi F, et al. Tumor Growth Assessment by Computed Tomography Perfusion Imaging (CTPI), Perfusion-Weighted Imaging (PWI), and Diffusion-Weighted Imaging (DWI) in a Rabbit Pleural Squamous Cell Carcinoma VX2-Implanted Model. Med Sci Monit. 2018;24:6756-6764. doi: 10.12659/MSM.909431

11. Tang Z, Chen F, Huang J, Shi L, Gong HT, Fu H, et al. Low-dose cerebral CT perfusion imaging (CTPI) of senile dementia: diagnostic performance. Arch Gerontol Geriatr. 2013;56(1):61-67. doi: 10.1016/j.archger.2012.05.009
12. Yang $\mathrm{Y}$, Zhu M, Wang $\mathrm{Y}$, Yang $\mathrm{H}, \mathrm{Wu} \mathrm{Y,} \mathrm{Li} \mathrm{B,} \mathrm{et} \mathrm{al.}$ Super-Resolution Reconstruction of Cell Pseudo-Color Image Based on Raman Technology. Sensors (Basel). 2019;19(19):4076. doi: 10.3390/s19194076

13. Jiang C, Zhang Q, Fan R, Hu Z. Super-resolution CT Image Reconstruction Based on Dictionary Learning and Sparse Representation. Sci Rep. 2018;8(1):8799. doi: 10.1038/ s41598-018-27261-z

14. Kaji S, Kida S. Overview of image-to-image translation by use of deep neural networks: denoising, super-resolution, modality conversion, and reconstruction in medical imaging. Radiol Phys Technol. 2019;12(3):235-248. doi: 10.1007/s12194-019-00520-y

15. Yao B, Li W, Pan W, Yang Z, Chen D, et al. Image reconstruction with a deep convolutional neural network in high-density super-resolution microscopy. Opt Express. 2020;28(10):15432-15446. doi: 10.1364/OE.392358

16. Sage D, Donati L, Soulez F, Fortun D, Schmit G, et al. DeconvolutionLab2: An open-source software for deconvolution microscopy. Methods. 2017; 115: 28-41. doi: 10.1016/j.ymeth.2016.12.015

17. Maria M, Arsalan M, Muhammad A, Nuzhat $H$. Analysis of renal artery morphometery in adults: a study conducted by using multidetector computed tomography angiography. Pak J Med Sci. 2017;33(4):943-947. doi: $10.12669 /$ pjms.334.13063

18. Rahman MM, Hun DC, Hussin S, Tzar MN, Ramli SR. A case of mycotic aneurysm due to burkholderia pseudomallei. Pak J Med Sci, 2013;29(2):666-668. doi: 10.12669 / pjms.292.2815

19. Chen F, Chen J, Peng Z, Jiang G, Yu M, et al. Virtual-view PSNR prediction based on a depth distortion tolerance model and support vector machine. Appl Opt. 2017; 56(30): 8547-8554. doi: 10.1364/AO.56.008547

20. Slupe AM, Kirsch JR. Effects of anesthesia on cerebral blood flow, metabolism, and neuroprotection. J Cereb Blood Flow Metab. 2018;38(12):2192-2208. doi: $10.1177 / 0271678 X 18789273$

\section{Authors Contribution:}

BF: Conceived the study, literature review, analysis of data and drafting the manuscript.

HZ: Takes the responsibility and is accountable for all aspects of the work in ensuring that questions related to the accuracy or integrity of any part of the work are appropriately investigated and resolved.
Authors:

1. Bo Fang, associate chief physician. Department of Neurology,

2. Hongjiang Zhai, chief physician. Department of Neurology,

1,2: Lu'an People's Hospital Affiliated to Anhui Medical University, Lu'an, 237005, Anhui Province, China. 